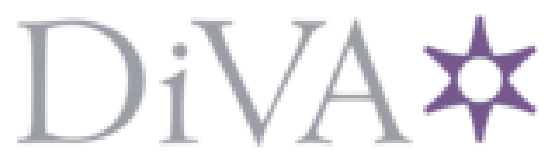

http://www.diva-portal.org

\title{
Preprint
}

This is the submitted version of a paper presented at IEEE International Conference on Communications (ICC) 2014,Sydney, Australia from 10-14 June 2014.

Citation for the original published paper:

Shokri-Ghadikolaei, H., Fischione, C. (2014)

Distributed random sensing order analysis and optimization in cognitive radio systems.

In: (pp. 1-1). IEEE Communications Society

N.B. When citing this work, cite the original published paper.

Permanent link to this version:

http://urn.kb.se/resolve?urn=urn:nbn:se:kth:diva-136465 


\section{Distributed Random Sensing Order Analysis and Optimization in Cognitive Radio Systems}

\author{
Hossein Shokri-Ghadikolaei \\ Automatic Control Department \\ Electrical Engineering and ACCESS \\ KTH Royal Institute of Technology \\ 10044, Stockholm, Sweden \\ hshokridieee.org
}

\author{
Carlo Fischione \\ Automatic Control Department \\ Electrical Engineering and ACCESS \\ KTH Royal Institute of Technology \\ 10044, Stockholm, Sweden \\ carlofiekth.se
}

\begin{abstract}
Developing an efficient spectrum access policy enables cognitive radios to dramatically increase spectrum utilization while assuring predetermined quality of service levels for the primary users. In this paper, modeling, performance analysis, and optimization of a distributed secondary network with random sensing order policy are studied. Specifically, the secondary users create a random order of the available channels and then find a transmission opportunity in a distributed manner. By a Markov chain analysis, the average throughputs of the secondary users and average interference level between the secondary and primary users are evaluated. Then, a maximization of the performance of the secondary network in terms of throughput while keeping under control the average interference is proposed. A simple and practical adaptive algorithm is established to optimize the network. Finally, numerical results are provided to validate the analytical derivations and demonstrate the performance of the proposed schemes. It is shown that distributed algorithms can achieve substantial performance improvements in cognitive radio networks without the need of centralized operations or management.
\end{abstract}

Keywords-Cognitive radio, sequential channel sensing, Markov model, average throughput, average interference.

\section{INTRODUCTION}

Emerging new wireless applications and ever-growing demands for a higher data rate challenges the limited spectrum resources and consequently the current fixed spectrum allocation policies. In order to effectively mitigate the problems associated with the fixed spectrum allocation policies, the promising concept of cognitive radio networks (CRNs) has been the focus of intense research in both academic and regulatory bodies [1].

CRN can promote spectrum utilization by allowing lowpriority secondary users (SUs) to opportunistically exploit the unused licensed channels of high-priority primary users (PUs). Meanwhile, due to preemptive priority of the PUs to access the channels, the SUs must vacate the channel whenever the corresponding PUs appear. In this case, a set of procedures called spectrum handoff ( $\mathrm{SHO}$ ) is initiated to help the SU to effectively find a new transmission opportunity and resume its unfinished transmission [2]. To this end, temporarily-available transmission opportunities must be explored first. Depending on the power budget and affordable computational complexity, an SU may sense one channel or multiple channels at a time [3]. We assume that the SUs can sense and possibly transmit on one channel at a time. In this case, an SU performs sequential channel sensing (SCS), meaning that it senses the first channel placed in its sensing order ( $\mathrm{SO}$ ) and then transmits on the channel provided that it is sensed free. If the channel is sensed busy, the SU initiates the SHO procedure and then senses the second channel of its SO. This kind of sensing-access is called sequential channel sensing [2].

In this paper, we focus on a slot-based SHO, meaning that the SUs and the PUs have slotted-based communications. The SUs sense the channels at the beginning of each time slot and initiate the SHO procedure whenever the current channel is sensed busy. In [4]-[6], performance of connectionbased SHO is extensively evaluated, and several SO setting policies are proposed. For the slot-based SHO, the optimal SO design has been investigated in [7] in order for an SU to achieve the maximum energy efficiency by applying a dynamic programming solution. Optimal and suboptimal SOs of a CRN containing only one SU are developed in [7][9], which maximize the average achievable throughput of the SU in a time slot. These results have been further extended for a CRN with two [10] and multiple SUs [11], [12]. In particular, a closed-form optimal solution as well as three suboptimal solutions for maximizing the average throughput through setting proper SOs have been proposed in [11], and an intelligent SO setting scheme for a centralized CRN has been introduced in [12].

However, most of the literatures in slot-based SHO focus on single SU or centralized CRNs [7]-[12]. A p-persistent MAC protocol has been introduced in [13], where the authors have applied the protocol for a distributed network operation. In [14], an autonomous SO setting strategy is proposed for distributed CRNs with the aim of minimizing the likelihood of collisions with other SUs. However, the miss detection probability is assumed zero, meaning that the SUs do not make interference for the PUs as well as other SUs. Therefore, QoS provisioning for the PUs is not addressed in that study. In [15], the authors exploit a modified p-persistent MAC protocol to set the SOs of the SUs in a distributed manner.

In this paper, we substantially extend our previous work [15], and investigate the performance of random sensing orders for the SUs. That is, once an SHO is triggered, all the SU create a set of random channels to be sensed. Then, the SCS process is initiated. We propose a finite state novel Markovian 
process-based structure to effectively model the SUs' behavior. Using this model, the performance of the random SO policy in terms of the average SUs' throughputs and average interference between the SUs and the PUs is derived. Based on this, a simple and practical adaptive algorithm is developed to iteratively maximize the average throughput while keeping the average interference bounded. Compared to the literature mentioned above, this is the first paper to 1) consider the problem of SHO for sequential channel sensing in a distributed set-up with multiple SUs with more realistic assumptions including miss detection and false alarm probabilities, 2) investigate the impact of the SUs' transmissions on the channel occupation probabilities, 3) derive the accumulated interference caused by other SUs' transmissions, 4) pose an optimization problem and develop a practical adaptive algorithm to maximize the average throughput while provisioning QoS for the PUs.

The rest of this paper is organized as follows. In Section II, we describe the considered CR network. In Section III, the structure of the random sensing order policy is described, and its performance is evaluated. Moreover, an efficient algorithm is proposed to optimize the performance of the network. Numerical results are then presented in Section IV, followed by concluding remarks provided in Section V.

\section{System Model}

A time slotted CRN with $N_{s}$ SUs is assumed. The SUs attempt to opportunistically transmit on the channels exclusively dedicated to the $N_{p}$ PUs, each having one channel. As assumed in [8]-[10], the SUs are synchronous in time-slots with other SUs as well as the PUs. In the SCS methodology, once a handoff is requested, each SU's time slot divides into sensing and transmission modes. In the sensing mode, the SUs sequentially sense the channels based on their SOs [7][12]. More specifically, it is assumed that the SUs sense the first channel of their SOs, they start their communications on the channels provided that they are sensed free. Other SUs initiate the handoff process, which takes $\tau_{h o}$ seconds ${ }^{1}$, and then sense the second channel of their SOs. The procedure continues until one of the following events happens [11]: a) transmission opportunities are found for all the SUs, b) no time remains for sensing new channels in the time slot, or c) no non-sensed channels are remained. After sensing $(n-1)$ occupied channels $n=1, \ldots, \delta$, where $\delta$ is a constant, if the SU finds the channel number $n$ free, the user will transmit data on that channel for the rest of the slot. If an SU starts transmitting on the channel $n$ its SO, the time length left in the slot for the transmission is

$$
R T_{n}=T-\tau-(n-1)\left(\tau+\tau_{h o}\right)
$$

where $T$ is a time slot duration, and $\tau$ is the channel sensing time.

The limited observations and dynamic nature of the wireless environment leads to imperfection in spectrum sensing, which are usually described by false alarm and miss detection probabilities, represented by $P_{f a, m}$ and $P_{m d, m}$ for sensing the channel $m$ for $m=1, \ldots, N_{p}$.

\footnotetext{
${ }^{1}$ The SU spends this time to change its circuitry in order to be prepared for sensing the next channel assigned to its SO. This time duration do not depend on the amount of frequency shift required by the reconfiguration [11].
}

\section{RAndom SEnsing ORder POLICY}

In this section, the random sensing order policy (RSOP) is introduced, and the performance of a CRN with RSOP is derived using a Markov chain analysis. Then, an adaptive protocol is proposed to optimize the performance of the CRN.

\section{A. Modeling}

Recall that each SU sequentially senses the channels based on an order. This order can be optimally determined in a centralized CRN [8], [11], wherein the SUs are placed in a list so that the average achievable throughput is maximized. However, those proposals cannot be directly applied to a distributed CRNs. For the networks, simple SOs are proposed in [15], wherein the channels are arranged by their indices. Suppose that the SO of the $n$-th SU is

$$
c_{n}=\left[c_{1 n}, c_{2 n}, \ldots, c_{\delta n}\right],
$$

where $c_{1 n}$ and $c_{\delta n}$ denote the first and the last channels to be sensed. $\delta$ is the maximum number of channels that can be sensed by an SU in a time slot. It holds [15] that

$$
\delta=1+\min \left(\frac{T-\tau}{\tau+\tau_{h o}}, N_{p}-1\right)
$$

For the simple SO, we have [15]:

$$
c_{1 j}=1 \quad c_{2 j}=2 \quad \ldots \quad c_{\delta j}=\delta, 1 \leq j \leq N_{s} .
$$

While this SO facilitates the network modeling and performance evaluation, a high level of contention to access the spectrum resources is imposed to the SUs, which significantly degrades the average throughput of the CRN.

In order to mitigate the aforementioned problem, the RSOP can be used. In this scheme, an SU randomly ${ }^{2}$ chooses a target channel in each sensing interval. That is, the SU randomly picks a value between 1 and $N_{p}$ for each $c_{i j}$. Therefore, the requests of the SUs are distributed among all available channels, and thereby the CRN throughput increases through the reduction of the contention level among the SUs. In order to further decrease the contention and provide multiple access among the SUs, a modified version of the conventional ppersistent multiple access protocol is utilized in this paper. That is, each SU senses each channel with the probability $p$ and skips the sensing process with the probability $(1-p)$. Channel sensing probability $p$, provides a degree of freedom to optimize the performance of the CRN in the form of maximizing the average throughput and keeping the interference bounded.

Fig. 1 models (by a Markov chain) the channel sensingaccess policy of the RSOP used by each SU. In this figure, the state $m^{(n)}$ refers to the case that the SU starts to sense, which takes $\tau$ time units, and possibly transmits on the channel $m$ at the $n$-th sensing stage. Let $P_{m, 0}^{(n)}$ and $P_{m, 1}^{(n)}$ respectively be the probability that the channel $m$ is free and occupied at the beginning of the $n$-th sensing stage. An SU can successfully transmit on the channel $m$, if it is free, and the false alarm does not occur. Once this event happens, with the probability of $P_{m, 0}^{(n)}\left(1-P_{f a, m}\right)$, the SU's state changes to the transmitter nodes $T_{n}$, and it transmits on the channel for the rest of the time slot, $R T_{n}$, with the constant rate of

\footnotetext{
${ }^{2}$ with uniform distribution
} 
$C_{R}$. Even though we consider constant transmission rate, the formulations presented in the paper can be easily extended to cover heterogenous SUs. This is a similar analysis assumption done in [10]. The interference experience at node $n$ is denoted by $I_{n}$, happens whenever the channel is busy, and the SU mistakenly senses it free, with probability $P_{m, 1}^{(n)}\left(1-P_{d, m}\right)$, where $P_{d, m}=1-P_{m d, m}$. Different handoffs are modeled using nodes $H O_{i}, i=1,2, \ldots, \delta$, where $\delta$ is defined in (3). Note that the first handoff node does not exist in the search process, and we use it for easing the analysis without loss of generality.

At the beginning of each time slot, an SU gives the state $\mathrm{HO}_{1}$ (in Fig. 1), and immediately transits to one of the first sensing nodes, $1^{(1)}, 2^{(n)}$, or $N_{p}{ }^{(1)}$ with the identical probabilities of $p / N_{p}$, or to the synchronizer state $S Y N 1^{3}$. After $\tau$ time units, the SU's state changes to the transmitter state (node $T_{1}$ ), interference state (node $I_{1}$ ), or to the second handoff node $\left(\mathrm{HO}_{2}\right)$ with the probability $q_{m}^{(1)}$. From Fig. 1,

$$
q_{m}^{(1)}=P_{m, 0}^{(1)} P_{f a, m}+P_{m, 1}^{(1)} P_{d, m} .
$$

This procedure continues until the maximum number of admissible handoff is reached. Let us define the $i$-th stage of the sensing-access process, shown in Fig. 1, as the set of nodes of the Markov chain $H O_{i}, S Y N_{i}, m^{(i)}, T_{i}$, and $I_{i}$. After $\delta$ stage, the SU's state transits to the terminate node $T E$, meaning that the SU sleeps for the rest of the time slot, $T-\tau-\delta\left(\tau+\tau_{h o}\right)$. Then, it repeats the search-access process at the beginning of the next slot [8]. In the RSOP, a busy channel can be occupied either by the corresponding PU or other SUs; because it may have been detected as a transmission opportunity by other SUs at the previous stages.

Proposition I: Consider the Markov Chain of Fig. 1. The occupation probability of channel $m$ at the beginning of stage $n$,

$$
P_{m, 1}^{(n)}=P_{m, 1}^{(n-1)}+P_{m, 0} P_{f a, m}{ }^{(1)}+L^{(2)}+\cdots+L^{(n-2)} U_{m}^{(n-1)},
$$

where $L^{(n)}$ is the number of SUs sensing channel $m$ at stage $n$, and $U_{m}^{(n)}$ is the probability of transmission on channel $m$ at stage $n$ by at least one SU conditioned on the absence of the corresponding PU.

Proof: The proof is given in Appendix A.

Proposition II: Consider the Markov Chain of Fig. 1. Let $T$ represent slot duration, $\mathcal{P}_{T_{n}, m}$ denote the probability of successful transmissions of each SU at each channel $m$ from node $T_{n}, R T_{n}$ be the remained time of the current slot, and $C_{R}$ indicate the constant transmission rate of the SUs. Let $\mathcal{B}_{I_{n}, m}$ be the probability that no SU cause interference on the channel $m$ at the stage $n$. Then, the average throughput of each SU is

$$
r=\frac{1}{T} \sum_{m=1}^{N_{p}} \sum_{n=1}^{\delta} \mathcal{P}_{T_{n}, m} R T_{n} C_{R}
$$

\footnotetext{
${ }^{3}$ Note that the SUs that are not routed to the sensing states, with the probability of $(1-p)$, enter standby mode (at node $S Y N_{1}$ ) and wait for $\tau$ time units (sensing period). Then, they are directed to the state $\mathrm{HO}_{2}$. With the help of the synchronizer nodes, all SUs will enter the $i$-th stage node at the same time.
}

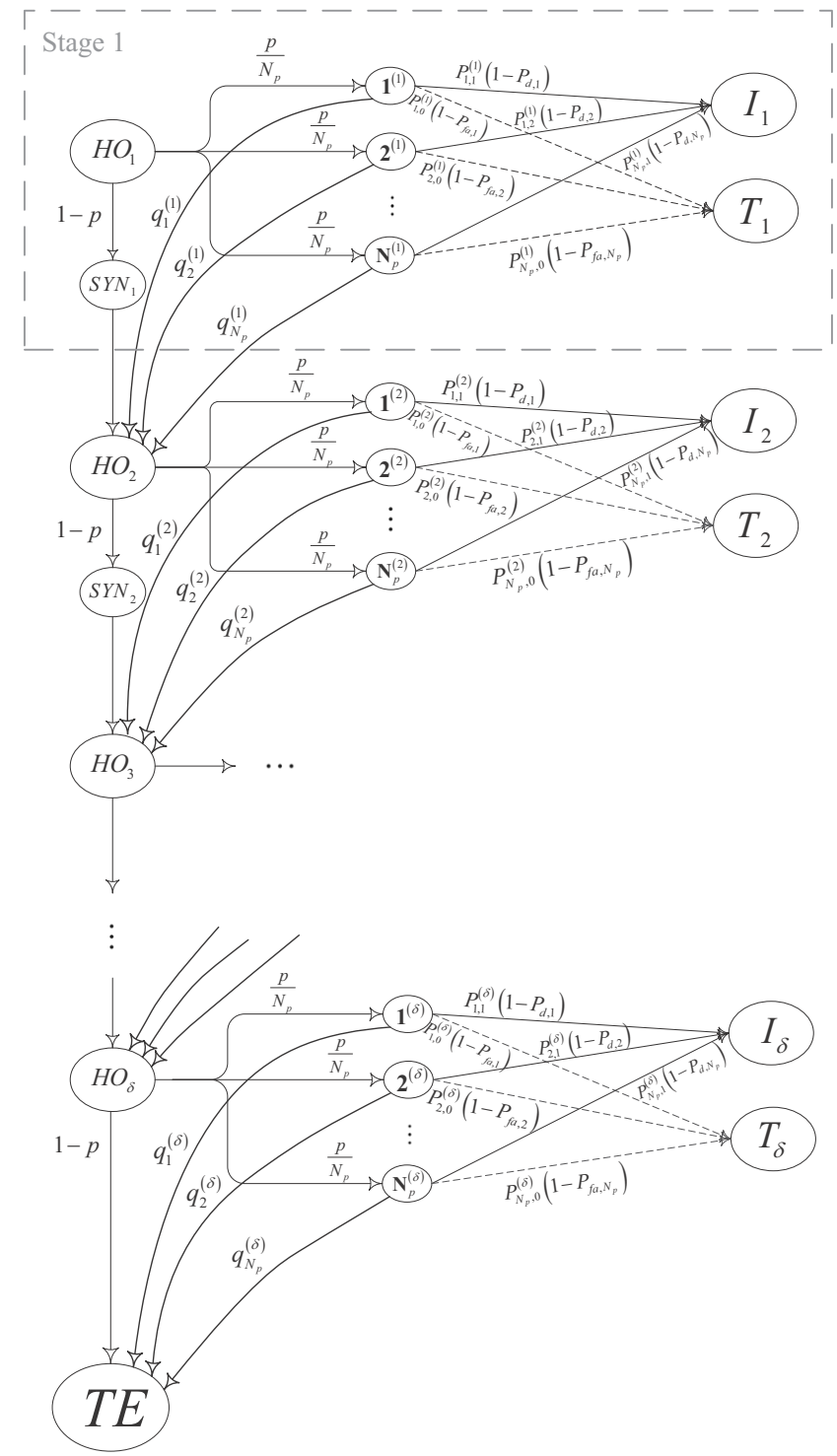

Fig. 1. Proposed Markov chain to model the SU's behavior. The states correspond to different stages of spectrum sensing and packet transmission.

Also, the average interference time due to the each SU's transmissions is

$$
t_{I}=\frac{1}{T N_{p}} \sum_{m=1}^{N_{p}} \sum_{n=1}^{\delta}\left(1-\mathcal{B}_{I_{n}, m}\right) R T_{n} .
$$

Proof: The proof is given in Appendix B.

Now that we have derived the key performance indicators, we can turn our attention to the optimization of the throughput.

\section{B. Performance optimization}

As can be observed from the previous two propositions, performance measures given by (7) and (8) depend on $\tau$ and $p$. Hence, the performance of the CRN can be optimized by choosing the values of $p$ and $\tau$ that maximize the average throughput, as a QoS metric for the SU, and bounding the 
interference time, as a QoS metric for the PUs as well as the SUs. That is,

$$
\begin{aligned}
& \tau^{*}=\operatorname{argmax} \quad r \\
& \begin{array}{ll} 
& \tau, p \\
\text { s.t. } & t_{I} \leq t_{I}^{\max }
\end{array} \\
& 0 \leq \tau \leq T
\end{aligned}
$$

where $t_{I}^{\max }$ represents the maximum tolerable value of the interference time, which depends on the QoS level guaranteed for the PUs as well as the SU. The optimization problem is generally non-convex, making it difficult to be efficiently solved for the global optimum. Also, due to the parameter $\delta$ in cost and constraint functions, both of them are not differentiable (see (3)). Hence, in the next subsection, we develop a novel efficient distributed algorithm to find a suboptimal solution for (9).

\section{Adaptive sequential channel sensing}

In the RSOP, some SUs enter the transmission or interference states at some stages like the $i$-th stage and consequently do not continue the search process among the channels. Therefore, in the average sense, less number of SUs further participate in the search process in the next stages, i.e., the $(i+1)$-th stage. Moreover, with a higher value for each channel sensing probability $p$, more SUs contend for accessing the channels. As a consequence, appropriately changing the channel sensing probability can lead to an increase in the achieved SUs throughput. From another perspective, as it is possible that some SUs transmit on occupied channels in each stage $i$, the remained SUs face a higher received signal levels if they sense those channels at the stage $i+1$. Therefore, in order to achieve the same sensing performance, $\tau$ can be decreased [16], and thereby the SUs will have more transmission time in the consecutive stages. It is worth mentioning that changing the average signal level that is present in the wireless media will affect $P_{m d, m}$. Note that the false alarm probability only depends on the noise level [16]. Clearly, with higher signal level, the miss detection probability is reduced. Hence, we take the worst case into account and assume that $P_{m d, m}$ is equal to the miss detection probability caused by the first sensing stage, and more precise modeling is left for future studies.

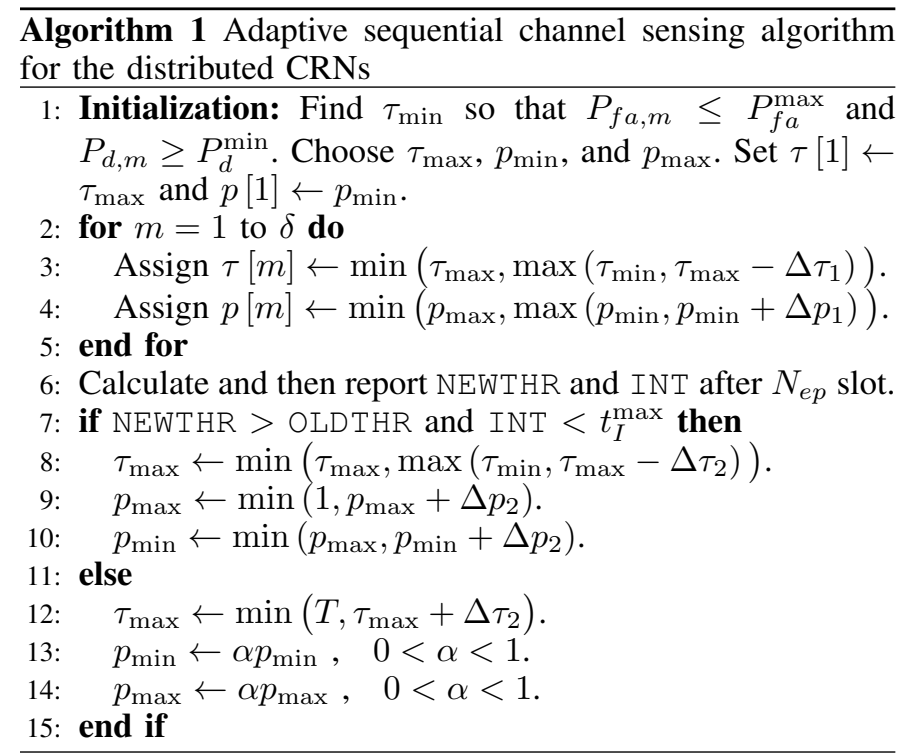

TABLE I. Simulation Parameters

\begin{tabular}{lll}
\hline \hline Parameter & Description & Value \\
\hline$T$ & Time slot duration & $10 \mathrm{~ms}$ \\
$P_{d}^{\min }$ & Minimum allowable detection probability & 0.9 \\
$P_{f a}^{\max }$ & Maximum allowable false alarm probability & 0.1 \\
$t_{I}^{\max }$ & Maximum allowable interference time & $0.05 \mathrm{~T}$ \\
$f_{s}$ & Receiver sampling frequency & $6.857 \mathrm{MHz}$ \\
$\tau_{h o}$ & Required time for performing a handoff & $0.1 \mu \mathrm{s}$ \\
$C R$ & Transmission rate & $1 \mathrm{bit} / \mathrm{s} / \mathrm{Hz}$ \\
\hline \hline
\end{tabular}

Let $p[n]$ and $\tau[n]$ denote the channel sensing probability and sensing time at stage $n$, and $p_{\min }, p_{\max }, \tau_{\min }$, and $\tau_{\max }$ are their minimum and maximum values. Also, $P_{f a}^{\max }$ and $P_{d}^{\min }$ represent the maximum allowable value for the false alarm and minimum of the detection probabilities announced by the CRN standard or other parties. We now allow the SUs to adjust the channel sensing probability as well as sensing time in each stages. We implement an additive-increase/multiplicativedecrease (AIMD) policy to let the SUs scale up their channel sensing probability and an additive-increase/additive-decrease (AIAD) policy for adjusting the sensing time in the consecutive stages. The algorithm starts with $p[1]=p_{\min }$ and $\tau[1]=\tau_{\max }$. Then, it linearly increases (decreases) the channel sensing probability (sensing time) of each SU in every stage. Meanwhile, the average throughput and interference are calculated for $N_{e p}$ slots [17], called NEWTHR and INT. Then, NEWTHR is compared with the old one which is computed in the previous estimation period, called OLDTHR. Once a reduction in the average throughput is detected, a congested channel is assumed, and consequently the transmitter decreases the transmission rate through reducing $p_{\min }$ and $p_{\max }$ and also increasing $\tau_{\max }$. Otherwise, the SU raises $p_{\min }$ and $p_{\max }$ and reduces $\tau_{\max }$ in order to enhance the transmission rate. The proposed distributed algorithm is summarized in Algorithm 1. It is clear that the nonadaptive protocol is a special case of the adaptive one with a fixed values for $p[m]=p, \tau[m]=\tau$, and a constant scaling factor $\alpha=1$.

\section{NumericAl Results}

In order to set up a simulation environment, we simulate an energy detector. The detailed structure of the energy detector can be found in [16]. The values of $P_{d}^{\min }, P_{f a}^{\max }$, time slot duration $T$, and the value of sampling frequency used by the energy detector, are chosen according to IEEE 802.22 standard [18]. Table I summarizes the descriptions and values of the parameters considered for the simulations. Using a Monte Carlo simulation, the average throughput and the average interference time are computed after simulating the scenarios for $10^{4}$ times.

Figs. 2 and 3 respectively depict the average throughput of the secondary network as well as the normalized interference time, versus channel sensing probability $p$ and normalized sensing time $\tau / T$. Clearly, as the channel sensing probability increases, the chance of finding a transmission opportunity (correctly or mistakenly) raises as well, leading to higher values for average throughput and interference. However, after an optimum point, the throughput reduces due to high connection level among the SUs. Moreover, by increment of the channel sensing time, the SUs sense the channels more accurately, find more transmission opportunity, and hence reach a higher average throughput. For the same reason, the average interference 


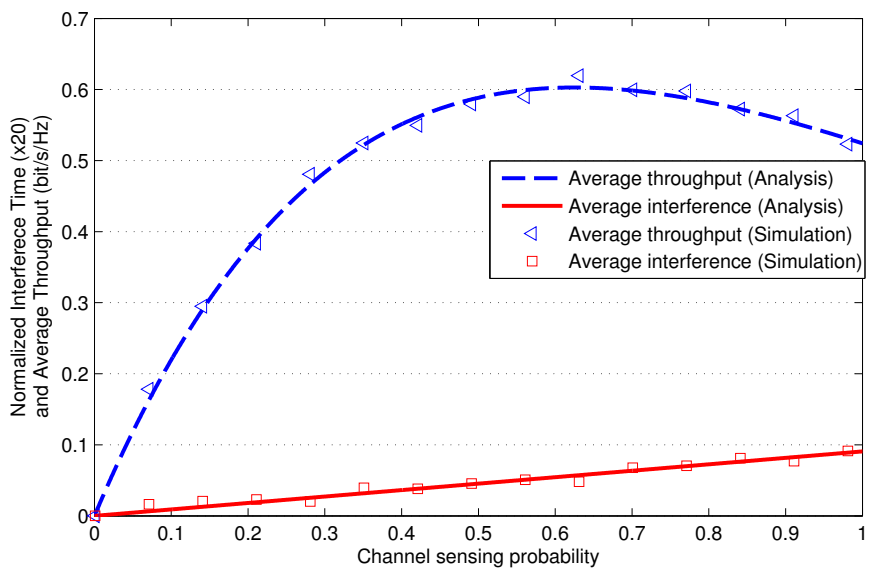

Fig. 2. Average throughput and interference against channel sensing probability (for $\tau=0.1 T, N_{s}=20$, and $N_{p}=5$ ).

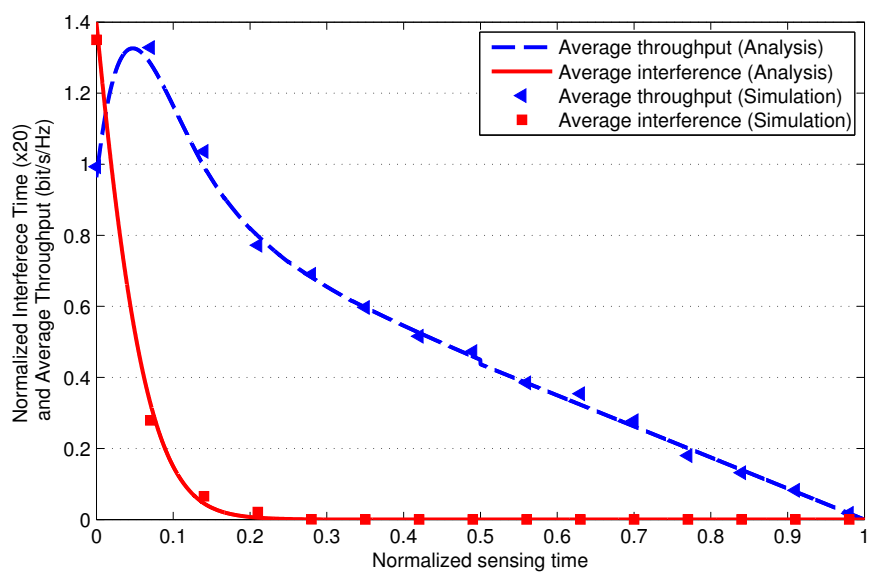

Fig. 3. Average throughput and interference against normalized sensing time (for $p=0.8, N_{s}=3$, and $N_{p}=7$ ).

time between the SUs and the PUs is reduced. Moreover, the well known sensing-throughput tradeoff [16] is verified. That is, after an optimum point, wherein the false alarm and miss detection probabilities are in acceptable levels, the average throughput starts decreasing due to the reduction of the time left for the transmission.

We simulate the adaptive sequential channel sensing with the following parameters: $N_{e p}=50, \tau_{\max }=0.5 T, \Delta \tau_{1}=$ $\Delta \tau_{2}=0.05 T, p_{\min }=0.5, p_{\max }=1, \Delta p_{1}=\Delta p_{2}=0.1$, and $\alpha=0.5$. It is worth noting that these initial values are just an example to illustrate the effectiveness of the proposed algorithm, and one can easily investigate the impact of the aforementioned parameters on the performance of the algorithm such as convergence rate and throughput enhancement rate, and then tries to adopt optimal initial values. Table II demonstrates the performance enhancement due to optimal $p$ and $\tau$ derived in (9), and compares the average throughput and interference for two different scenarios: (1) optimal values, which are obtained by a brute force numerical optimization search and (2) adaptive values as achieved by our proposed Algorithm 1. As expected, adopting the optimal and adaptive values for $p$ and $\tau$ increases the average throughput while the interference meets the constraint. Specifically, for the case
TABLE II. AVERAge throughput (Avg. Thr.) AND THE CORRESPONDING NORMALIZED INTERFERENCE (NOR. INT.), FOR THE OPTIMAL PARAMETERS AND ADAPTIVE PARAMETERS.

\begin{tabular}{c|c|c|c|c}
\cline { 2 - 5 } & \multicolumn{2}{c|}{ Optimal Parameters } & \multicolumn{2}{c}{ Adaptive Parameters } \\
\cline { 2 - 5 } & Avg. Thr. & Nor. Int. & Avg. Thr. & Nor. Int. \\
\hline \hline$N_{s}=3, N_{p}=7$ & 1.6742 & 0.0470 & 1.6292 & 0.0422 \\
\hline$N_{s}=5, N_{p}=7$ & 1.9349 & 0.0500 & 1.9007 & 0.0489 \\
\hline$N_{s}=7, N_{p}=3$ & 1.2986 & 0.0500 & 1.2830 & 0.0473 \\
\hline$N_{s}=7, N_{p}=5$ & 1.7655 & 0.0429 & 1.7099 & 0.0480 \\
\hline
\end{tabular}

$N_{s}=3, N_{p}=7$, the average throughput of the SUs achieved by the optimal design respectively is about $44 \%$ and $2.7 \%$ more than the ones achieved in $p=0.8, \tau=0.1 T$ (see Fig. 3) and adaptive algorithm. The exhaustive search benefits the maximum throughput at the cost of a huge computational burden. From the table, we see that the average throughput raises with the number of primary channel $N_{p}$. This is due to that more channels are sensed, and therefore more transmission opportunities are found, which results in higher average throughput. A similar pattern can be observed between the average interference and the number of the SUs.

\section{CONCLUSiON}

Modeling and performance evaluation of random sensing order policy (RSOP) in a distributed cognitive radio network $(\mathrm{CRN})$ were investigated in this paper. The behaviors of the secondary users (SUs) were modeled through a novel Markov process. The performance of the RSOP in terms of the average throughput of the CRN and average interference levels between the SUs and the primary users was evaluated. Then, an optimization problem was formulated to maximize the average throughput while the interference level is kept bounded. Finally, to enhance the RSOP performance, a simple but efficient algorithm was proposed to adaptively adjust the sensing-access parameters. This algorithm enhances the performance of the CRN without high computational burden, as demonstrated through exhaustive numerical performance evaluations.

\section{APPENDIX A}

Let $P_{m, 1}^{(n)}$ denote the occupation probability of the $m$-th channel at the beginning of the $n$-th stage. At the beginning of the first stage, the SUs have not sensed any channels yet, and therefore the occupation probability of each channel is equal to the corresponding PU's presence probability ${ }^{4}$. Thus, we have

$$
P_{m, 1}^{(1)}=P_{m, 1}, \quad 1 \leq m \leq N_{p}
$$

Let $\mathcal{N}_{x}$ be the number of the SUs that have requests at the node $x$. So, from Fig. 1 , we have, $\mathcal{N}_{H O_{1}}=N_{s}$. The average number of the SUs that sense the $m$-th channel at the first stage, represented by $L^{(1)}$, can be computed as

$$
L^{(1)}=\mathcal{N}_{m^{(1)}}=\frac{p}{N_{p}} \mathcal{N}_{H O_{1}}=p \frac{N_{s}}{N_{p}} .
$$

Each channel $m$ is sensed by $L^{(1)}$ SUs at the first stage. Each of these SUs might sense the corresponding channel free.

\footnotetext{
${ }^{4}$ These probabilities can be determined by modeling the PUs activities, e.g., well-known ON-OFF model [4].
} 
In this case, the user starts its transmission on the channel, and therefore contributes to this channel's occupation probability. The probability of transmission on the $m$-th channel by at least one SU conditioned on the absence of the PU is,

$$
U_{m}^{(1)}=1-P_{f a, m}^{L^{(1)}}
$$

$U_{m}^{(n)}$ is the probability that at least one SU transmits on the $m$-th channel (or equivalently one SU senses the channel free) at the end of the $n$-th stage conditioned on the absence of the PU. Considering (10), (11), and (12), we have

$$
\begin{gathered}
P_{m, 1}^{(2)}=P_{m, 1}+P_{m, 0} U_{m}^{(1)} \\
\mathcal{N}_{H O_{2}}=(1-p) \mathcal{N}_{H O_{1}}+\sum_{m=1}^{N_{p}} q_{m}^{(1)} \mathcal{N}_{m}^{(1)} \\
=\left((1-p)+\frac{p}{N_{p}} \sum_{m=1}^{N_{p}} q_{m}^{(1)}\right) \mathcal{N}_{H O_{1}} .
\end{gathered}
$$

At the second stage, the number of SUs whose requests enter the node $\mathrm{HO}_{2}$ is calculated in (14), where $q_{m}^{(n)}$ is defined in (5). We have,

$$
U_{m}^{(2)}=1-P_{f a, m}^{L^{(2)}}
$$

where $L^{(2)}=\left(p / N_{p}\right) \mathcal{N}_{\mathrm{HO}_{2}}$. Therefore, the $m$-th channel occupation probability at the beginning of the third stage can be computed as:

$$
\begin{aligned}
P_{m, 1}^{(3)} & =P_{m, 1}+P_{m, 0} U_{m}^{(1)}+P_{m, 0} P_{f a, m}^{L^{(1)}} U_{m}^{(2)} \\
& =P_{m, 1}^{(2)}+P_{m, 0} P_{f a, m}^{L^{(1)}} U_{m}^{(2)}
\end{aligned}
$$

Following the same steps, at the $n$-th stage we have,

$$
P_{m, 1}^{(n)}=P_{m, 1}^{(n-1)}+P_{m, 0} P_{f a, m}^{L^{(1)}+L^{(2)}+\cdots+L^{(n-2)}} U_{m}^{(n-1)}
$$

where

$$
\begin{aligned}
U_{m}^{(n)} & =1-P_{f a, m}^{L^{(n)}} \\
L^{(n)} & =\frac{p}{N_{p}} \mathcal{N}_{H O_{n}}
\end{aligned}
$$

and

$$
\mathcal{N}_{H O_{n}}=\left[(1-p)+\frac{p}{N_{p}} \sum_{m=1}^{N_{p}} q_{m}^{(n-1)}\right] \mathcal{N}_{H O_{n-1}}
$$

\section{APPENDIX B}

Let $\Pi_{x}$ denote the steady state probability of being at the state $x$. From Fig. 1, we have,

$$
\begin{gathered}
\Pi_{H O_{n}}=\left[(1-p)+\frac{p}{N_{p}} \sum_{m=1}^{N_{p}} q_{m}^{(n-1)}\right] \Pi_{H O_{n-1}} \\
\Pi_{m^{(n)}}=\frac{p}{N_{p}} \Pi_{H O_{(n)}} \\
\Pi_{I_{n}}=\sum_{m=1}^{N_{p}} P_{m, 1}^{(n)}\left(1-P_{d, m}\right) \Pi_{m^{(n)}}
\end{gathered}
$$

and

$$
\Pi_{T_{n}}=\sum_{m=1}^{N_{p}} P_{m, 0}^{(n)}\left(1-P_{f a, m}\right) \Pi_{m^{(n)}} .
$$

Note that considering the channel search and access policy described in Section III, the procedure always initiates from the node $H O_{1}$, and thus

$$
\Pi_{H O_{1}}=1 .
$$

Then, from (20)-(23), $\Pi_{I_{n}}$ and $\Pi_{T_{n}}$ are calculated. Let $P_{T_{n}, m^{(n)}}^{[k]}$ and $P_{I_{n}, m^{(n)}}^{[k]}$ respectively denote the probability of the transmissions from the nodes $T_{n}$ and $I_{n}$ on the channel $m$, i.e., the state changes from the node $m^{(n)}$ to the nodes $T_{n}$ and $I_{n}$ :

$$
\begin{gathered}
P_{T_{n}, m^{(n)}}^{[k]}=\Pi_{m^{(n)}}^{[k]} P_{m, 0}^{(n)}\left(1-P_{f a, m}\right) \\
P_{I_{n}, m^{(n)}}^{[k]}=\Pi_{m^{(n)}}^{[k]} P_{m, 1}^{(n)}\left(1-P_{d, m}\right)
\end{gathered}
$$

The $k$-th SU will be able to successfully transmit data on each channel $m$ at the stage $n$ (with the probability $\mathcal{P}_{T_{n}, m}^{[k]}$ ) provided that its state transits to from the nodes $m^{(n)}$ to $T_{n}$ for $1 \leq n \leq \delta$ (with the probability $P_{T_{n}, m^{(n)}}^{[k]}$ ), and all other SUs do not ever collide its communications. Assume that $\mathcal{A}_{m}^{[\ell]}$ represents the probability of the SU $\ell$ does not transmit on the channel $m$ in a time slot. Hence, the $k$-th SU successfully transmits data on the channel $m$ at the stage $n$ with the probability of

$$
\mathcal{P}_{T_{n}, m}^{[k]}=\prod_{\substack{\ell=1 \\ \ell \neq k}}^{N_{s}} P_{T_{n}, m^{(n)}}^{[k]} \mathcal{A}_{m}^{[\ell]}
$$

If we omit the superscript $[k],(27)$ is simplified to $\mathcal{P}_{T_{n}, m}=$ $P_{T_{n}, m^{(n)}} \mathcal{A}_{m}^{N_{s}-1}$. At the stage, the SU transmits for $R T_{n}$ time units with the constant rate of $C_{R}$. The average throughput of each SU is computed:

$$
r=\frac{1}{T} \sum_{m=1}^{N_{p}} \sum_{n=1}^{\delta} \mathcal{P}_{T_{n}, m} R T_{n} C_{R}
$$

Finally, we need to formulate $\mathcal{A}_{m}$. To this end, the proposed Markov model is modified in which regardless of the stage, the SU cannot send on the channel $m$. Fig. 4 depicts the $n$-th stage of the pruned Markov model. As can be observed, if the SU enters to the state $m^{(n)}$, it will be immediately routed to the next handoff state. Using this figure and following the same steps taken in the Appendices A and B, the steady states probabilities of being at $T_{n}, I_{n}$ or eventually $T E$ is obtained. Then, we have,

$$
\mathcal{A}_{m}=\Pi_{T E}+\sum_{n=1}^{\delta} \Pi_{T_{n}}+\Pi_{I_{n}} .
$$

In order to find the average interference time, $t_{I}$, note that each SU encounters

$$
t_{I}^{[k]}=\frac{1}{T N_{p}} \sum_{n=1}^{\delta} \Pi_{I_{n}} R T_{n}
$$




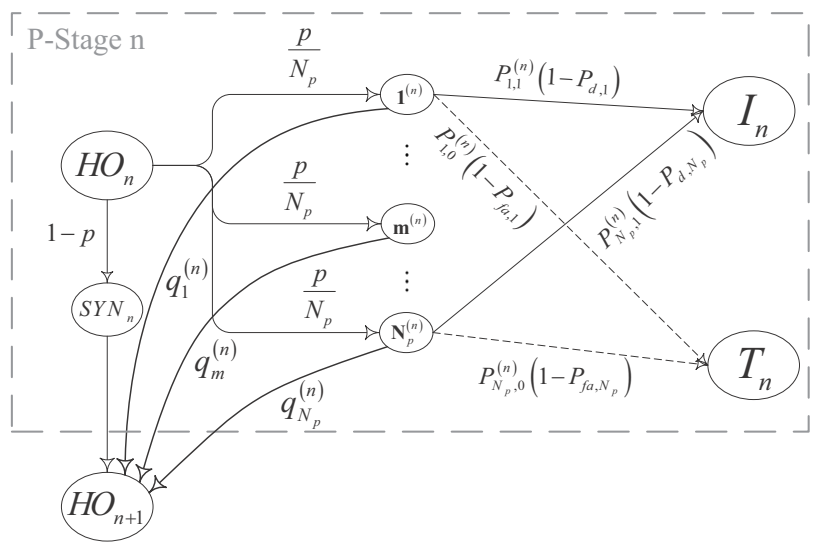

Fig. 4. Pruned Markov model.

level of interference in each time slot. But, these random variables are not independent; because the $\mathrm{SU} k_{2}$ can transmit on the occupied channel $m$, where it has been mistakenly interfered by the SU $k_{1}$ in the previous sensing stages. Therefore,

$$
\frac{1}{T N_{p}} \sum_{k=1}^{N_{s}} \sum_{n=1}^{\delta} \Pi_{I_{n}} R T_{n}
$$

is an upper bound of the interference time of the network. Let $\mathcal{B}_{I_{n}, m}$ be the probability no SUs cause interference on the channel $m$ at the stage $n$. From (26), we have,

$$
\mathcal{B}_{I_{n}, m}=\prod_{k=1}^{N_{s}}\left(1-P_{I_{n}, m^{(n)}}^{[k]}\right)=\left(1-P_{I_{n}, m^{(n)}}\right)^{N_{s}} .
$$

Hence, the average interference time of the network is calculated as:

$$
t_{I}=\frac{1}{T N_{p}} \sum_{m=1}^{N_{p}} \sum_{n=1}^{\delta}\left(1-\mathcal{B}_{I_{n}, m}\right) R T_{n}
$$

\section{ACKNOWLEDGMENTS}

The authors are supported by the EU projects Hycon2 and Hydrobionets.

\section{REFERENCES}

[1] J. Wang, M. Ghosh, and K. Challapali, "Emerging cognitive radio applications: A survey," IEEE Commun. Mag., vol. 49, no. 3, pp. 74-81, Mar. 2011.

[2] I. Christian, S. Moh, I. Chung, and J. Lee, "Spectrum mobility in cognitive radio networks," IEEE Commun. Mag., vol. 50, no. 6, pp. 114-121, June 2012

[3] H. Sun, A. Nallanathan, C. Wang, and Y. Chen, "Wideband spectrum sensing for cognitive radio networks: a survey," IEEE Wireless Com munications, vol. 20, no. 2, pp. 74-81, Apr. 2013.

[4] L. Wang, C. Wang, and C. Chang, "Modeling and analysis for spectrum handoffs in cognitive radio networks," IEEE Trans. on Mobile Comput., vol. 11, no. 9, pp. 1499-1513, Sept. 2012.

[5] _ - "Optimal target channel sequence design for multiple spectrum handoffs in cognitive radio networks," IEEE Trans. on Commun., vol. 6 , no. 1, pp. 2444-2455, Sept. 2012.
[6] C. Zhang and K. Shin, "What should secondary users do upon incumbents' return?" IEEE J. on Sel. Areas in Commun., vol. 31, no. 3, pp. 417-428, Mar. 2013.

[7] Y. Pei, Y. Liang, K. Teh, and K. Li, "Energy-efficient design of sequential channel sensing in cognitive radio networks: optimal sensing strategy, power allocation, and sensing order," IEEE J. on Sel. Areas in Commun., vol. 29, no. 8, pp. 1648-1659, Sept. 2011.

[8] H. Jiang, R. F. L. Lai, and H. V. Poor, "Optimal selection of channel sensing order in cognitive radio," IEEE Trans. Wireless Commun., vol. 8, no. 1, pp. 297-307, Jan. 2009.

[9] Z. Zhang and H. Jiang, "Cognitive radio with imperfect spectrum sensing: The optimal set of channels to sense," IEEE Wireless Commun. Letters, vol. 1, no. 2, pp. 133-136, Apr. 2012.

[10] R. Fan and H. Jiang, "Channel sensing-order setting in cognitive radio networks: A two-user case," IEEE Trans. Veh. Technol., vol. 58, no. 9, pp. 4997-5008, Nov. 2009.

[11] H. Shokri-Ghadikolaei and M. Nasiri-Kenari, "Sensing matrix setting schemes for cognitive networks and their performance analysis," IET Commun., vol. 6, no. 17, pp. 3026-3035, Nov. 2012.

[12] H. Shokri-Ghadikolaei and R. Fallahi, "Intelligent sensing matrix setting in cognitive radio networks," IEEE Commun. Letters, vol. 16, no. 11, pp. 1824-1827, Nov. 2012.

[13] L. Lai, H. E. Gamal, H. Jiang, and H. V. Poor, "Cognitive medium access: Exploration, Exploitation, and Competition," IEEE Trans. Mobile Computing, vol. 10, no. 2, pp. 239-253, Feb. 2011.

[14] Z. Khan, J. Lehtomaki, L. DaSilva, and M. Latva-aho, "Autonomous sensing order selection strategies exploiting channel access information," IEEE Trans. on Mobile Comput., vol. 12, no. 2, pp. 274-288, Feb. 2013.

[15] H. Shokri-Ghadikolaei, F. Sheikholeslami, and M. Nasiri-Kenari, "Distributed multiuser sequential channel sensing schemes in multichannel cognitive radio networks," IEEE Trans. Wireless Commun., vol. 12, no. 5, pp. 2055-2067, May 2013.

[16] Y. C. Liang, Y. Zeng, E. C. Y. Peh, and A. T. Hoang, "Sensingthroughput tradeoff for cognitive radio networks," IEEE Trans. Wireless Communication, vol. 7, no. 4, pp. 1326-1337, Apr. 2008.

[17] S. H. Park, H. Yoon, and J. W. Kim, "A cross-layered network adaptive $\mathrm{HD}$ video steaming in digital A/V home network: Channel monitoring and video rate adaptation," IEEE Trans. on Consum. Electron., vol. 52, no. 4 , pp. 1245-1252, Nov. 2006.

[18] "Draft standard for wireless regional area networks part 22: Cognitive wireless RAN medium access control (MAC) and physical layer (PHY) specifications," IEEE 802.22 Working Group. 